\section{P4 ARE ROUTINE CHEST X-RAYS NECESSARY IN ASYMPTOMATIC PATIENTS NEWLY DIAGNOSED WITH HIV?}

doi:10.1136/sextrans-2012-050601c.4

${ }^{1} \mathrm{M}$ L Cheshire, ${ }^{*} \mathrm{C}$ Babu. ${ }^{1}$ Manchester Medical School, Manchester, UK; ${ }^{2}$ Manchester Centre for Sexual Health, Manchester, UK

Background This audit looked at the use of chest-X-rays (CXRs) in newly diagnosed HIV patients at an inner-city GUM clinic. The Departmental guidelines recommended that all newly diagnosed HIV patients had a baseline CXR. The 2011 British HIV Association guidelines (which were under consultation at the time of the audit) proposed that CXRs should only be done in patients with current or previous chest disease, high-risk for TB or in intravenous drug users. Aims The aims of this audit were twofold, first to confirm whether all newly diagnosed patients had a CXR as per departmental guidelines, and second to review the results of the CXR in order to see whether, if any, factors predicted abnormal results.

Methods The audit considered all newly diagnosed HIV patients from 1 April 2009-31 March 2011. Data including demographic details, past medical history, health on diagnosis and details of the CXR (if performed) were collected from electronic HIV summaries and radiology records. Statistical analysis was performed using SPSS. Results A total of 196 patients were identified, 69\% of whom had had a CXR. In those who had a CXR and in whom results were available $(\mathrm{n}=132), 92 \%$ had a normal CXR and $8 \%$ abnormal. Significant predictors of abnormal CXRs included chest symptoms at diagnosis $(p<0.001)$ and a CD4 count $<200(p=0.001)$. There was no significant link with the patients' country of origin but there was a pattern of association which was clinically relevant.

Conclusions In this audit all CXRs in asymptomatic patients newly diagnosed with HIV were normal. No latent chest disease was identified as a result of routine asymptomatic screening with CXRs and the practice is not justified. Following this audit clinic guidelines have been appropriately amended in keeping with current British HIV Association guidelines with the additional criteria for performing CXRs in patients presenting with CD4 $<200$.

\section{P5 CHANGING TRENDS IN HIV DIAGNOSIS IN AN INNER CITY LONDON TEACHING HOSPITAL BETWEEN 2007 AND 2011}

doi:10.1136/sextrans-2012-050601c.5

${ }^{1} \mathrm{H}$ Wilkin-Crowe, ${ }^{2} \mathrm{R}$ Lau, ${ }^{*}{ }^{2} \mathrm{M}$ Pakianathan. ${ }^{1}$ St. George's Medical School, London, UK; ${ }^{2}$ St. George's Healthcare NHS Trust, London, UK

Background In 2008, guidelines were published by the British Association for Sexual Health and HIV, British HIV Association and British Infection Society recommending normalisation of HIV testing to reduce stigma and to increase the number of early diagnoses. The guidelines aimed at reducing morbidity, mortality and reducing the risk of onward transmission of HIV. Local initiatives were carried out in South West London to achieve this by extensive general practice education programmes to raise HIV testing awareness in non-sexual health ( $\mathrm{SH}$ ) settings.

Aims To establish the effect of a number of local initiatives to promote HIV testing in non-SH settings at a large South West London hospital.

Methods Data on all new diagnoses referred to the HIV outpatient were collected and analysed from electronic patient records, including ethnodemographic data, place of diagnosis and clinical data including baseline CD4 count. Previously diagnosed patients having received HIV care elsewhere transferring into the service were excluded. Fisher's exact t-test was used for statistical analysis.
Results and discussion There were 394 patients with a new diagnosis of HIV between 2007 and 2011. Diagnosis by primary care and other non-SH settings increased by $184 \%$. There was an increase of $37 \%$ in the median CD4 count at diagnosis, taking the median count to 372 cells $/ \mathrm{mm}^{3}$ in 2011, showing the majority of patients to have been diagnosed at an early stage. The proportion of very late presenters decreased by $24 \%$.

Conclusions The evidence suggests that local initiatives are successfully implementing National guidelines. More work is needed to increase HIV testing and reduce the number of late diagnoses.

\section{P6 CULTURAL DIFFERENCES IN THE ACCEPTABILITY OF HOME SAMPLING FOR HIV INFECTION}

doi:10.1136/sextrans-2012-050601c.6

${ }^{1} \mathrm{C}$ A Bowman, ${ }^{1} \mathrm{G}$ Bell, ${ }^{1} \mathrm{H}$ Ellam, ${ }^{1} \mathrm{~A}$ Tunbridge, ${ }^{2} \mathrm{~S}$ Slack, ${ }^{2} \mathrm{~A}$ Atkin, ${ }^{1} \mathrm{C}$ Evans, ${ }^{2} \mathrm{~A}$ Shutt, ${ }^{2}$ E Joseph, ${ }^{1} S$ Naylor, ${ }^{1} S$ Herman, ${ }^{1} S$ Green, ${ }^{1} G$ Kudesia, ${ }^{1} A$ Pryce. ${ }^{*}{ }^{1}$ Royal Hallamshire Hospital, Sheffield, UK; ${ }^{2}$ Centre for HIV and Sexual Health, Sheffield, UK

Background MSM community outreach using oral home sampling kits posted to virology for testing previously demonstrated success in attracting non-healthcare seeking individuals at risk of HIV. The outcome of targeting other specific at-risk groups to offer home sampling has not previously been described.

Objective To determine the acceptability of home sampling kits for HIV using oral swabs in two at-risk groups Black Africans (BA) and partners of HIV positive patients (PPP).

Methods Self-taken oral fluid home sampling kits were returned to virology for testing using two HIV assays: Roche COBAS and Genscreen Ultra (previously validated for oral fluid testing). Total IgG was also measured to assess sample adequacy. Participant recruitment was two-pronged: community based (BA) or via an HIV clinic (PPP). For BA recruitment, home sampling kits were actively promoted at relevant social events and venues by trained African volunteers from July to December 2010. 19 free condom distribution points were also utilised to provide information about HIV and the testing kits. From September to December 2011, PPPs of unknown current HIV status were contacted and offered the option of attending clinic or receiving an oral fluid home sampling kit by post. Results Despite intense promotional activity, only 12 kits from 11 individuals in the BA community project were returned: 5 male; 6 female. Two of these participants were not African. In the PPP clinic based study, of 46 partners offered a kit, 38 (83\%) accepted, and $34(89 \%)$ returned a sample. BA partners were less likely to accept a home sampling kit $(9 / 13 ; 69 \%)$ than white partners $(29 / 33$; $88 \%)$ in the PPP group. Participant feedback was favourable in both studies.

Discussion Further evaluation is needed to understand the difference in acceptability of this method of HIV testing in specific at-risk groups (MSM, BA and PPP) in community and clinic settings.

\section{P7 A WORLD RECORD FOR HIV TESTING. PUBLICITY STUNT OR EFFECTIVE OUTREACH?}

doi:10.1136/sextrans-2012-050601c.7

J Jenkins, ${ }^{*}$ A G McOwan, S McCormack, L J Chislett. Chelsea and Westminster Hospital NHS Foundation Trust, London, UK

Background 1 in 7 MSM in London are HIV positive, 1 of 5 of whom are unaware of this because they have never tested, or acquired HIV since their last test. Our service took the opportunity presented by World AIDS Day to promote the importance of regular HIV testing in MSM by staging a HIV testing world record attempt in a local gay bar. 